\title{
Verification of Piecewise Linear Systems using Abstractions
}

\author{
Valur Einarsson, Torkel Glad \\ Department of Electrical Engineering \\ Linköpings universitet, SE-581 83 Linköping, Sweden \\ WWW: http://www. control.isy.liu.se \\ Email: $\{$ valure, torkel\}@isy.liu.se
}

February 23, 1999

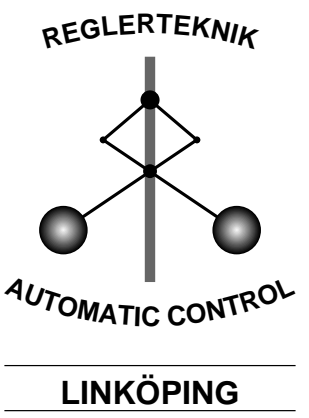

Report no.: LiTH-ISY-R-2112

Technical reports from the Automatic Control group in Linköping are available by anonymous ftp at the address ftp.control.isy.liu.se. This report is contained in the compressed postscript file 2112 .ps. Z. 


\title{
Verification of Piecewise Linear Systems using Abstractions
}

\author{
Valur Einarsson, Torkel Glad \\ Division of Automatic Control, \\ Department of Electrical Engineering, \\ Linköping University, S-581 83 Linköping, Sweden. \\ E-mail: \{valure, torkel\}@isy.liu.se \\ URL: http://www.control.isy.liu.se
}

February 23, 1999

\begin{abstract}
A modeling framework for the class of piecewise linear switched systems is presented. This is done combining classical ordinary differential equations (ODEs) and logic. Methods for abstracting away from the details of ODEs using conservative discrete approximations are discussed and DEDS methods are used for verifying specifications. A fairly complex example is treated, the main result being that fully automated verification can be conclusive for models in this class.
\end{abstract}

\section{Introduction}

The topic of hybrid systems deals with modeling, analysis and control of systems where behavior is a combination of continuous evolution and abrupt changes. Traditionally, systems displaying purely continuous or purely discrete changes have been considered separately by people from control systems and computer science, respectively.

Continuous systems are most commonly modeled using differential equations. A variety of analysis and design methods exist, ranging from simulation to automatic controller synthesis, with simpler classes of systems allowing more advanced methods. The same relation holds for discrete event systems, however several modeling formalisms are commonly used, e.g. automata theory and Petri nets, each imposing a tradeoff between modeling capability and analysis or design methods.

A special class of hybrid systems are switched systems, where system trajectories are continuous but may have discontinuous derivatives. We intend to focus on this class here, and in particular those systems where the dynamics are governed by piecewise linear differential equations. Furthermore, the analysis consists of verifying properties of these systems against a given specification.

Piecewise linear systems have been treated, for instance, in [5] where graphical analysis methods are developed, and in [6] where several results are provided for the discrete time case. Other special classes have been treated recently, e.g., in [1] where systems with piecewise linear trajectories are considered, and in 
[2] where systems which can be modeled as timed automata are treated. An overview of general hybrid systems can, for instance, be obtained in [3].

The theory presented here originates from [8]. However, several modifications are introduced making it more suitable for automated verification.

\section{Modeling}

The hybrid systems considered are composed of three main components: a continuous time plant, a discrete event controller, and an interface. The interface provides communication between the plant and the controller by converting signals from the continuous domain of the former to the discrete, symbolic domain of the latter, and vice versa. The interface can be further decomposed into an actuator translating controller symbols to plant input signals and a generator transforming continuous signals to discrete symbols used by the controller.

The modeling framework is depicted in Figure 1. The controller reads the discrete output $\tilde{e}$ indicated by the generator and passes a new control symbol $\tilde{u}$ to the actuator. The actuator produces input signal $u(t)$ to the plant which in its turn affects the evolution of the continuous state $x(t)$.

We adopt the notation of [8] for distinguishing between signals and symbols and use tildes to indicate a symbol valued set or sequence. For example, we denote by $x(t)$ (or simply $x$ ) a continuous time signal, while $\tilde{x}$ is to be interpreted as a symbol. A more detailed description of the components in our framework

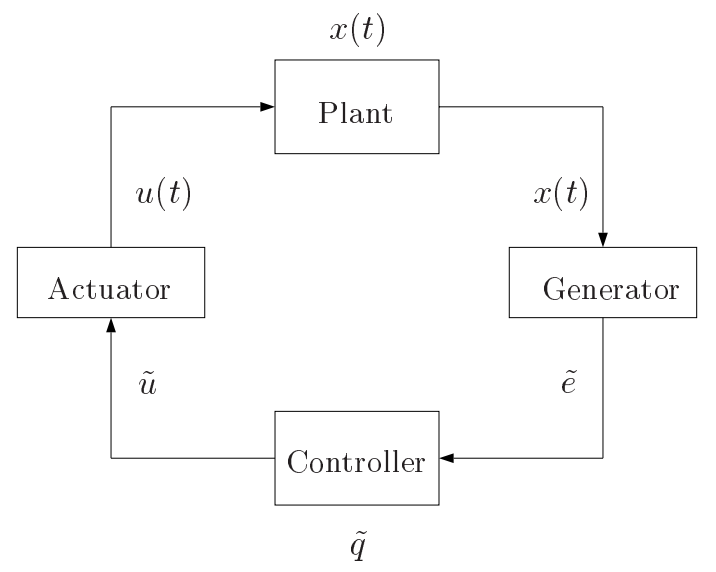

Figure 1: System configuration

follows.

The plant is continuous and time invariant, with dynamics piecewise affine in the continuous states and polynomial in the continuous inputs. More formally, we have the plant dynamics given by

$$
\dot{x}(t)=A(u(t)) x(t)+b(u(t))
$$

where $x(t) \in \mathbb{R}^{n}$ is the continuous state vector and $u(t) \in \mathbb{R}^{m}$ is a vector of input signals. Furthermore, the elements of the input vector $u$ enter the $n \times n$ matrix $A(u)$ and the $n \times m$ vector $b(u)$ polynomially. Note that although we refer to the continuous part of the system as the plant, it may also contain some purely continuous controllers. 
The controller is purely logical and modeled as a deterministic automaton (Moore machine), $C=\left\{\tilde{Q}, \tilde{q}_{0}, E, \tilde{k}, \tilde{U}, \tilde{l}\right\}$ where $\tilde{Q}$ is a set of controller states and $\tilde{q}_{0} \in \tilde{Q}$ is an initial state, $\tilde{E}$ is a set of controller input symbols and $\tilde{k}: \tilde{Q} \times \tilde{E} \rightarrow \tilde{Q}$ is the controller state transition function, $\tilde{q}^{+}=\tilde{k}(\tilde{q}, \tilde{e})$. Furthermore, $\tilde{U}$ is a set of controller output symbols and $\tilde{l}: \tilde{Q} \rightarrow \tilde{U}$ is the controller output function, $\tilde{u}=\tilde{l}(\tilde{q})$. We use Boolean vectors to encode the states and symbols, i.e., $\tilde{Q} \subseteq \mathbb{B}^{d}, \tilde{E} \subseteq \mathbb{B}^{p}$ and $\tilde{U} \subseteq \mathbb{B}^{m}$.

The actuator is a mechanism for applying continuous control signals $u(t)$ in accordance to the discrete control symbols $\tilde{u}$. The most straightforward manner to accomplish this is translating the Boolean constant true to the real constant 1 and the Boolean constant false to the real constant 0 ,

$$
\begin{aligned}
\tilde{u}_{i}=\text { true } \Rightarrow u_{i}(t) & =1 \\
\tilde{u}_{i}=\text { false } \Rightarrow u_{i}(t) & =0
\end{aligned}
$$

where $1 \leq i \leq m$.

The generator is a mechanism for relating the discrete symbols $\tilde{e}$ to the continuous signals, $x(t)$. The discrete symbols are generated using a set of $n-1$ dimensional hyperplanes, $\mathcal{H}_{i}=\left\{x \mid c_{i}^{T} x-d_{i}=0\right\}$. Plant events occur when the continuous trajectory enters or leaves a hyperplane, i.e., when $x\left(t^{-}\right) \notin \mathcal{H}_{i}$ and $x(t) \in \mathcal{H}_{i}$ or when $x\left(t^{-}\right) \in \mathcal{H}_{i}$ and $x(t) \notin \mathcal{H}_{i}$. In connection with an event, an output symbol is generated according to

$$
\tilde{e}_{i}= \begin{cases}\text { true } & \text { if } x(t) \in \mathcal{H}_{i}, x\left(t^{-}\right) \notin \mathcal{H}_{i} \\ \text { false } & \text { otherwise }\end{cases}
$$

The above definitions are illustrated in the following example.

Example Consider the controlled water tank in Figure 2. We assume that

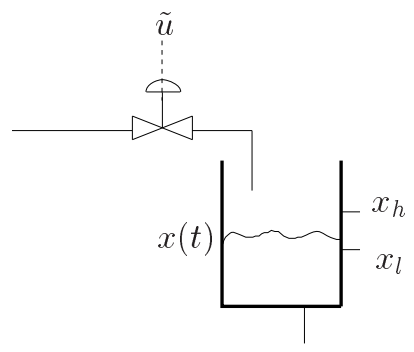

Figure 2: A water tank

the tank is linear, i.e., we can obtain a linearization which is valid for the whole continuous region. This leads to the plant model

$$
\dot{x}=a x+b u
$$

where $a, b$ are constants. In this one dimensional example, the generator consists of two points, hence

$$
\begin{array}{r}
\mathcal{H}_{1}=\left\{x \mid x-x_{l}=0\right\} \\
\mathcal{H}_{2}=\left\{x \mid x-x_{h}=0\right\}
\end{array}
$$


Thus, the event $\tilde{e}=$ (true, false) corresponds to passing the low level indicator while $\tilde{e}=($ false, true) represents going from normal level to high level or from high level to normal. The controller has the states on and off, represented by $\tilde{q}=$ true and $\tilde{q}=$ false, respectively, and the controller input is the event vector $\tilde{e}$. The controller is designed to keep the level between high and low, this is accomplished using the transition function according to Table 1 . The

\begin{tabular}{|c|c||cc|}
\hline \multicolumn{2}{|c||}{$\tilde{k}(\tilde{q}, \tilde{e})$} & \multicolumn{2}{c|}{$\tilde{e}$} \\
\hline \hline \multirow{2}{*}{$\tilde{q}$} & false & true, false) & (false, true $)$ \\
& true & true & false \\
& true & false \\
\hline
\end{tabular}

Table 1: Controller transition function

controller output is simply $\tilde{l}(\tilde{q})=\tilde{q}$ and we can define the initial controller state as $\tilde{q}_{0}=$ true. The resulting controller automaton is shown in Figure 3.

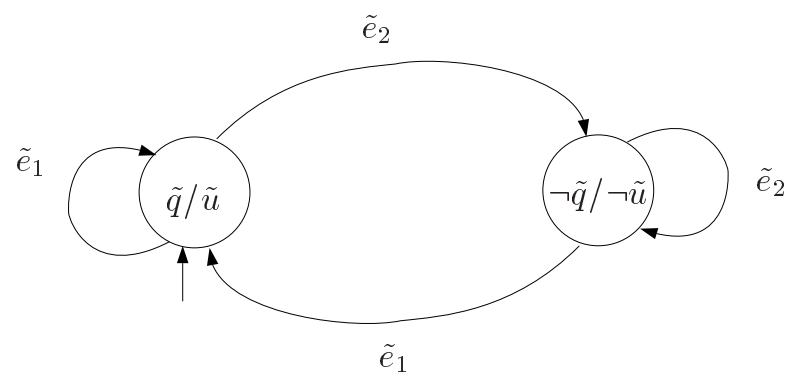

Figure 3: The controller automaton

Note that in the above example, we introduced hysteresis by allowing the controller to have discrete dynamics. This is always possible and provides a convenient way of modeling single sided constraints.

\section{Verification}

The model obtained in the previous section provides a compact description of the system and could, for instance, be analyzed by simulation. Another analysis method is formal verification, or model checking, frequently applied in the context of Discrete Event Dynamical Systems (DEDS). In order to apply those methods to our model, we need to obtain a discretization which conserves the properties we are interested in.

\subsection{DEDS Model}

We now intend to obtain a discrete model which approximates our combination of plant, generator and actuator. Since we will use the discrete model for verification we need to make sure that the approximation is conservative, i.e., that relevant properties of the discrete model also hold for the original hybrid model. 
We may therefore obtain different discrete models in order to verify different properties.

As before, we use the automaton formalism, defining the DEDS plant model as a nondeterministic automaton (Mealy machine), $P=\left\{\tilde{X}, \tilde{x}^{0}, \tilde{U}, \tilde{f}, \tilde{E}, \tilde{h}\right\}$ where $\tilde{X}$ is a set of plant states and $\tilde{x}^{0} \in \tilde{X}$ is an initial state, $\tilde{U}$ is a set of plant input symbols and $\tilde{f}: \tilde{X} \times \tilde{U} \rightarrow 2^{\tilde{X}}$ is the plant state transition function, $\tilde{x}^{+} \in \tilde{f}(\tilde{x}, \tilde{u})$. Furthermore, $\tilde{E}$ is a set of plant output symbols and $\tilde{h}: \tilde{X} \times \tilde{X} \rightarrow \tilde{E}$ is the plant output function, $\tilde{e}=\tilde{h}\left(\tilde{x}, \tilde{x}^{+}\right)$. As before, symbols are encoded using Boolean vectors, i.e., $\tilde{U} \subseteq \mathbb{B}^{m}$ and $\tilde{E} \subseteq \mathbb{B}^{p}$. However, we will find it convenient to assign one out of three values to the discrete state vector, i.e., $\tilde{X} \subseteq\{-1,0,1\}^{p}$.

The set of plant states is defined by the partition imposed by the generator through the set of hyperplanes. We associate each element of the discrete state vector with the two open halfspaces $\mathcal{H}_{i}^{-}=\left\{x \mid c_{i}^{T} x-d_{i}<0\right\}$ and $\mathcal{H}_{i}^{+}=$ $\left\{x \mid c_{i}^{T} x-d_{i}>0\right\}$, as well as the hyperplane $\mathcal{H}_{i}$ separating them, according to

$$
\begin{aligned}
x \in \mathcal{H}_{i}^{-} & \Rightarrow \tilde{x}_{i}=-1 \\
x \in \mathcal{H}_{i} & \Rightarrow \tilde{x}_{i}=0 \\
x \in \mathcal{H}_{i}^{+} & \Rightarrow \tilde{x}_{i}=1
\end{aligned}
$$

where $1 \leq i \leq p$. Thus, we obtain a partition of the continuous state space into a collection of open polyhedra, $\mathcal{P}_{\tilde{x}}=\left\{x \mid\left(c_{i}^{T} x-d_{i}\right) \tilde{x}_{i}>0, \tilde{x}_{i} \neq 0\right\} \cap\left\{x \mid c_{i}^{T} x-d_{i}=\right.$ $\left.0, \tilde{x}_{i}=0\right\}$ with an autonomous affine continuous system associated with each polyhedron. The set of discrete states then corresponds to the union of these polyhedra.

Definition 1 The set of DEDS plant states is defined as $\tilde{X}=\left\{\tilde{x} \mid \mathcal{P}_{\tilde{x}} \neq \emptyset\right\}$

We now need to define the transition relation for our discrete approximation. We are interested in two kinds of approximations. The first variant results in a discrete event dynamic system where impossible transitions are also impossible in the switched system. The second gives a DEDS where one of the possible transitions is guaranteed to occur in the switched system. We refer to the former as an outer approximation since it allows more behavior than the original system, and the latter as an inner approximation since it allows less behavior. Common to both types of transitions is the fact that we don't allow more than one transition at a time. This restricts transitions to ones from a $k$-dimensional polyhedron to one of its $k$-1-dimensional facets or vice versa. A corresponding relation thus holds for the involved discrete states.

Definition 2 Two discrete states $\tilde{x}^{1}, \tilde{x}^{2} \in \tilde{X}$ are called outward adjacent if they correspond to a $k$-dimensional polyhedra and one of its $k-1$-dimensional facets, i.e., if

$$
\overline{\mathcal{P}}_{\tilde{x}^{1}} \cap \mathcal{P}_{\tilde{x}^{2}} \neq \emptyset \quad \text { and } \quad \operatorname{dim} \mathcal{P}_{\tilde{x}^{1}}-\operatorname{dim} \mathcal{P}_{\tilde{x}^{2}}=1
$$

where $\overline{\mathcal{P}}$ denotes the closure of $\mathcal{P}, \overline{\mathcal{P}}=\mathcal{P} \cup \partial \mathcal{P}$. Analogously, we call the pair $\tilde{x}^{2}, \tilde{x}^{1} \in \tilde{X}$ inward adjacent states.

In addition to adjacency, the dynamics of the underlying continuous system must dictate that a transition can take place. This is where the two types of approximations differ. 
In the case of an outer approximation, the continuous dynamics of the corresponding region must allow for a transition to take place. This results in the following conditions.

Proposition 1 An outer transition from $\tilde{x}$ to $\tilde{x}^{+}$, where $\tilde{x}, \tilde{x}^{+} \in \tilde{X}$ are outward adjacent, is enabled, i.e., $\tilde{x}^{+} \in \tilde{f}^{o}(\tilde{x}, \tilde{u})$ provided that there exists a control symbol and a point on the facet such that the vector field makes an acute angle with the facet normal. That is,

$$
\exists \tilde{u} \in \tilde{U}, \exists x \in \mathcal{P}_{\tilde{x}+} \text { s.t. } c_{j}^{T}(A(\tilde{u}) x+b(\tilde{u})) \tilde{x}_{j}<0
$$

where $\tilde{x}_{j}^{+} \neq \tilde{x}_{j}$ and $A(\tilde{u})$ and $b(\tilde{u})$ are evaluated in an obvious manner based on the actuator relation (2). The conditions when the two states are inward adjacent are analogous.

Proof: Consider a trajectory, $x(t)$, such that at time $t, x(t) \in \mathcal{P}_{\tilde{x}^{+}}$and $\dot{x}(t)=A(\tilde{u}) x(t)+b(\tilde{u})$ for some $\tilde{x}, \tilde{x}^{+} \in \tilde{X}$ and $\tilde{u} \in \tilde{U}$. Due to outward adjacency we know that $x(t) \in \overline{\mathcal{P}}_{\tilde{x}}$ and since $\dot{x}(t)$ is directed from $\mathcal{P}_{\tilde{x}}$, we know that $x\left(t^{-}\right) \in \mathcal{P}_{\tilde{x}}$.

In the case of an inner approximation, the continuous dynamics of the corresponding polyhedron must guarantee that a transition takes place through one of the facets. This means that we need to ensure that $x(t)$ cannot be prevented from reaching the facets which allow transitions. This is provided by the following conditions.

Proposition 2 A sufficient condition for the state trajectory to exit a polyhedron, $\mathcal{P}_{\tilde{x}}$, is that there exists a bounded facet, $\mathcal{P}_{\tilde{x}^{\prime}}$, where $\tilde{x}, \tilde{x}^{\prime} \in \tilde{X}$ are outward adjacent, such that for all control symbols and all points in the closure of $\mathcal{P}_{\tilde{x}}$, the vector field makes an acute angle with the facet normal. That is,

$$
\forall \tilde{u} \in \tilde{U}, \forall x \in \overline{\mathcal{P}}_{\tilde{x}}, c_{j}^{T}(A(\tilde{u}) x+b(\tilde{u})) \tilde{x}_{j}<0
$$

where $\tilde{x}_{j}^{\prime} \neq \tilde{x}_{j}$. The transitions from such a polyhedron are called inner transitions, i.e., $\tilde{x}^{+} \in \tilde{f}^{i}(\tilde{x}, \tilde{u})$ when the above holds. The conditions for inward adjacent states are similar, the difference being that the angle condition now must hold at all points on the facet. That is

$$
\forall \tilde{u} \in \tilde{U}, \forall x \in \mathcal{P}_{\tilde{x}}, c_{j}^{T}(A(\tilde{u}) x+b(\tilde{u})) \tilde{x}_{j}^{\prime}>0
$$

where $\tilde{x}_{j} \neq \tilde{x}_{j}^{\prime}$.

Proof: In the outward adjacent case, we construct a scalar measure of the distance from a point in the polytope $\mathcal{P}_{\tilde{x}}$ to the hyperplane containing the facet $\mathcal{P}_{\tilde{x}^{\prime}}, V(x)=\tilde{x}_{j}\left(c_{j}^{T} x-d_{j}\right)$ where the index $j$ is determined by $\tilde{x}_{j}^{+} \neq \tilde{x}_{j}$. Clearly $V(x)$ is zero for points on the hyperplane and positive otherwise. We now examine how this distance varies as time evolves,

$$
\dot{V}(x)=\frac{d V(x(t))}{d t}=\tilde{x}_{j} c_{j}^{T} \dot{x}=\tilde{x}_{j} c_{j}^{T}(A(\tilde{u}) x+b(\tilde{u}))
$$

Since our condition guarantees that $\dot{V}(x) \leq-\epsilon<0$ for all $x \in \mathcal{P}_{\tilde{x}}, \tilde{u} \in \tilde{U}$, we know that the trajectory will eventually either reach the facet or it will leave the 
polytope via another facet. The inward adjacent case uses an argument similar to that of Proposition 1.

Finally, we define the output function. It is identical for the two types of approximations and simply assigns the value true to the element corresponding to the hyperplane being entered.

Definition 3 Let $\tilde{x}, \tilde{x}^{+}$be two states which satisfy the transition conditions of either Proposition 1 or Proposition 2. Then

$$
\tilde{h}_{i}\left(\tilde{x}, \tilde{x}^{+}\right)= \begin{cases}\text {true } & \text { if } \tilde{x}_{i}^{+}=0, \tilde{x}_{i} \neq 0 \\ \text { false } & \text { otherwise }\end{cases}
$$

Let us apply these ideas to the example from the previous section.

Example The discrete state space consists of 5 states, three corresponding to the open intervals where the level is low, normal or high, and two corresponding to the separating points. The former are encoded by $\tilde{x}^{1}=(-1,-1)^{T}, \tilde{x}^{3}=$ $(1,-1)^{T}$ and $\tilde{x}^{5}=(1,1)^{T}$ respectively, while the latter are represented by $\tilde{x}^{2}=$ $(0,-1)^{T}$ and $\tilde{x}^{4}=(1,0)^{T}$. Starting with the outer approximation, we see that without restrictions on control, it is always possible to make a transition to an adjacent state. It turns out that this is also the case for an inner approximation, provided that $-\frac{b}{a}>x_{h}$. Since the two approximations coincide, our abstraction is merely a different view of the system states and dynamics. The output is generated according to (16) and we may, for instance, select our initial state as $\tilde{x}^{0}=\tilde{x}^{3}=(1,-1)^{T}$. The resulting automaton is shown in Figure 4 where the

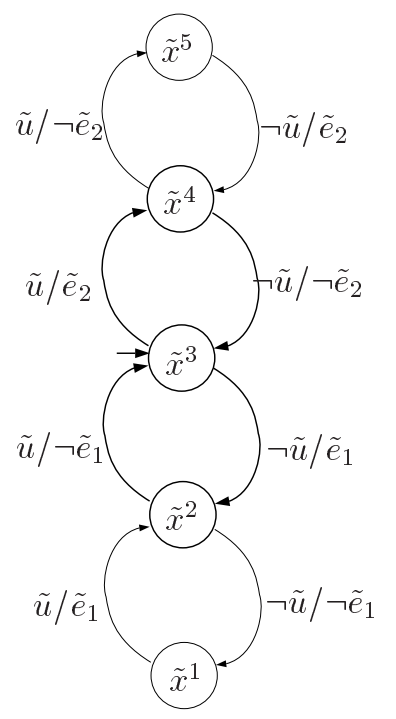

Figure 4: The tank automaton

transitions admitted by the controller and the states reachable under control are emphasized.

The example shows that as the plant makes a transition, it outputs events which in their turn force the controller to change its state. In contrast, the 
controller output, associated only with its state, acts as an enabling mechanism for plant transitions. This distinction between passive and active variables resembles the condition/event framework of [7] and provides a notion of causality.

Note that though the outer and inner approximations were identical in this example, this is not the case in general. However, due to the way we have constructed the approximations, we can use them for verifying certain properties of the original model. In the case of an outer approximation, guaranteeing that certain states are not reachable from an initial state in the discrete model implies that they are not reachable in the original model. Similarly, for an inner approximation, guaranteeing that a certain state is reachable from an initial state in the discrete model, irrespective of non-deterministic choices made, implies that the original model will eventually reach that state. Thus the former is suitable for examining safety properties while the latter can be used for planning.

Having obtained an appropriate discretization of our model, we can perform the verification on the discrete model. The core of DEDS verification is reachability analysis where the set of states reachable from a given set of initial states is obtained. Using symbolic representation of our discrete model, this can be done efficiently for quite complex systems [4].

\section{An Example}

As an example of how the methods described can be applied, we consider a model of the fictional chemical reaction process in Figure 5. Given a model of

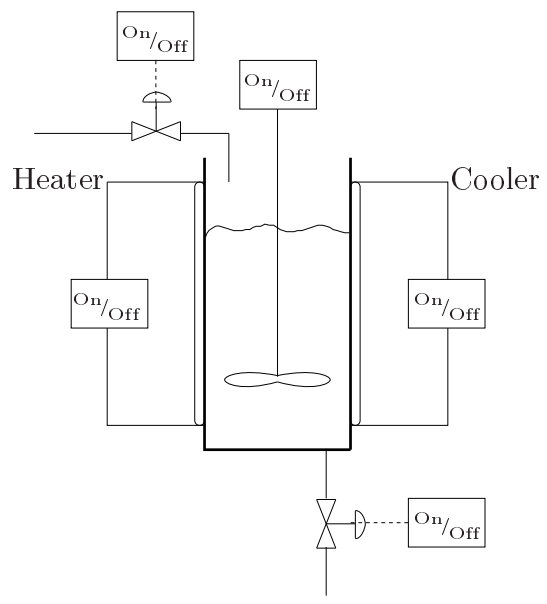

Figure 5: A chemical reactor

the reactor and a controller design, we wish to verify that certain properties hold. 


\subsection{Hybrid Model}

Denoting the level by $x_{1}$ and the temperature by $x_{2}$, the system can be described by (1) with

$$
\begin{aligned}
& A(u)=\left(\begin{array}{cc}
-a_{h} u_{d} & 0 \\
0 & -\left(a_{T_{1}}\left(1-u_{b}\right)+a_{T_{2}} u_{b}\right)
\end{array}\right) \\
& b(u)=\left(\begin{array}{c}
b_{h} u_{i} \\
b_{\text {heat }} u_{h}+b_{\text {cool }} u_{c}+b_{\text {reac }} u_{r}
\end{array}\right)
\end{aligned}
$$

where the continuous control signals $u_{*}$ can take values 0 (off) and 1 (on).

The controller has the output $\tilde{u}=\left(\tilde{u}_{b}, \tilde{u}_{i}, \tilde{u}_{d}, \tilde{u}_{h}, \tilde{u}_{c}, \tilde{u}_{r}\right)^{T}$ and its design is obtained using the following heuristics:

- Blender, $\tilde{u}_{b}$ : The blender is off when the fluid level is very low and on otherwise.

- Inflow, $\tilde{u}_{i}$ : The inflow valve is open while the fluid level is not high, then it is closed. It stays closed while the fluid level is not low.

- Draining, $\tilde{u}_{d}$ : The drain valve is closed when there is no reaction and open otherwise.

- Heater, $\tilde{u}_{h}$ : The heater is on when there is no reaction and off otherwise.

- Cooler, $\tilde{u}_{c}$ : The cooler is off while the temperature is not high, then it is turned on. It stays on while the temperature is not low.

- Reaction, $\tilde{u}_{r}$ : This variable indicates that the reaction has started, although it is a property of the system it is treated as an (uncontrollable) control variable.

Although we know how we want the controller to behave, we still have to define what we mean by "high level", "low temperature" etc. We thus need to design the interface between the logic controller and the continuous states, i.e., the generator.

The variables defining the discrete states of the system, and examples of their interpretation, are listed in Table 2. Note that the definition of "low" and

\begin{tabular}{|c|c|}
\hline variable, $\tilde{x}_{i}$ & $\tilde{x}_{i}=1$ \\
\hline$\tilde{x}_{1}$ & tank empty \\
$\tilde{x}_{2}$ & level very low \\
$\tilde{x}_{3}$ & level low \\
$\tilde{x}_{4}$ & level high \\
$\tilde{x}_{5}$ & tank full \\
$\tilde{x}_{6}$ & temperature below min \\
$\tilde{x}_{7}$ & no reaction \\
$\tilde{x}_{8}$ & temperature low \\
$\tilde{x}_{9}$ & temperature high \\
$\tilde{x}_{10}$ & temperature above max \\
\hline
\end{tabular}

Table 2: Discrete state variables

"high" fluid level depends on the temperature, the idea is that high fluid level may be less desirable at higher temperatures. 
The events generated force the controller to update its state according to

$$
\tilde{k}(\tilde{q}, \tilde{e})=\left(\begin{array}{rl}
\neg \tilde{e}_{2} \wedge \tilde{q}_{1} \vee \tilde{e}_{2} \wedge \neg \tilde{q}_{1} \\
\tilde{e}_{3} \wedge \neg \tilde{e}_{4} \vee \tilde{q}_{2} \wedge \neg \tilde{e}_{3} \wedge \neg \tilde{e}_{4} \\
\neg \tilde{e}_{7} \wedge \tilde{q}_{3} \vee \tilde{e}_{7} \wedge \neg \tilde{q}_{3} \\
\neg \tilde{e}_{\bar{e}} \wedge \tilde{q}_{4} \vee \tilde{e}_{7} \wedge \neg \tilde{q}_{4} \\
\tilde{e}_{9} \wedge \neg \tilde{e}_{8} \vee & \vee \tilde{q}_{5} \wedge \neg \tilde{e}_{9} \wedge \neg \tilde{e}_{8} \\
\neg \tilde{e}_{7} \wedge \tilde{q}_{6} \vee \tilde{e}_{7} \wedge \neg \tilde{q}_{6}
\end{array}\right)
$$

and the output is defined using $\tilde{l}(\tilde{q})=\left(\tilde{q}_{1}, \tilde{q}_{2}, \tilde{q}_{3}, \tilde{q}_{4}, \tilde{q}_{5}, \tilde{q}_{6}\right)^{T}$

An example trajectory obtained by simulating the hybrid model defined above is shown in Figure 6 along with the partition of the state space according to the generator. This trajectory might confirm with our ideas of normal op-

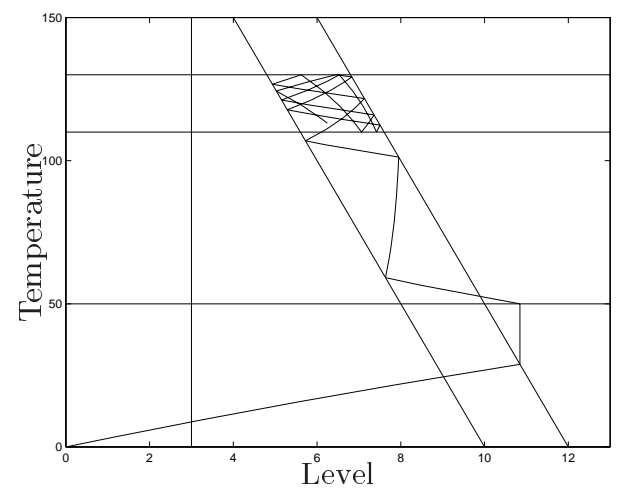

Figure 6: An example trajectory

eration, i.e., we would like the trajectory to gradually approach the operating region without exceeding allowed values on the way. We'll now try to be more specific about what we actually want and examine if this is achieved.

\subsection{Verification Results}

We start by giving a verbal specification of some properties which the controlled system should have:

1. The controller should guarantee that the temperature is never above maximum.

2. The controller should guarantee that the tank is never empty and that it never overflows.

3. There shall exist an operating region where the temperature is neither high nor low and the fluid level is neither high nor low. This region should be stable, i.e., once entered it should not be left under normal operating conditions.

4. The operating region should always be reachable from the initial states.

Properties 1 and 2 can be verified by obtaining the set of states which are backward reachable from the forbidden regions, using the outer approximation, and 
checking whether this set intersects the set of initial states. Property 4 can be checked using the outer approximation as well, starting in the operating region and ensuring that the forward reachable set is the operating region. The third property relies on the inner approximation and requires forward reachability analysis from the initial states. Here we consider the initial state when the level is very low but the tank is not empty, and the temperature is low but not below minimum. As a result, we can ensure that the properties stated hold for our hybrid model.

It should be emphasized that the steps towards our conclusions are fully automated, i.e., given a hybrid model we perform the verification without any further interference. This of course becomes more valuable as the dimension and complexity of the models increases.

\section{Conclusions}

We have presented a modeling framework for the class of piecewise linear switched systems, combining classical ordinary differential equations (ODEs) and logic. Methods for abstracting away from the details of ODEs, and thus allowing DEDS methods for verifying specifications, have been discussed. A fairly complex example has been treated using the methods described, the main result being that fully automated verification can be conclusive for models in this class.

Further work aims at using symbolic methods throughout the verification process. This provides means for dealing with complexity issues and enables parameterization of the controller/interface design and thus opens a window towards controller synthesis.

\section{References}

[1] R. Alur, C. Courcoubetis, N. Halbwachs, T. Henzinger, P.-H. Ho, X. Nicollin, A. Olivero, J. Sifakis, and S. Yovine. The algorithmic analysis of hybrid systems. Theoretical Computer Science, 138:3-34, 1995.

[2] R. Alur and D. Dill. A theory of timed automata. Theoretical Computer Science, 126(2):183-235, 1994.

[3] M. S. Branicky. General hybrid dynamical systems: Modeling, analysis, and control. In R. Alur, T. Henzinger, and E. D. Sontag, editors, Hybrid Systems III Verification and Control, volume 1066 of Lecture Notes in Computer Science, pages 186-200. Springer-Verlag, Berlin, 1996.

[4] J.R. Burch, E.M. Clarke, K.L. McMillan, D. Dill, and L.J.Hwang. Symbolic model checking: $10^{20}$ states and beyond. In Proc. of the 5th IEEE Symp. on Logic in Computer Science, pages 428-439, Philadelphia, PA, USA, June 1990. IEEE Computer Society Press, Los Alamitos, CA, USA.

[5] N. B. O. L. Pettit, T. Manavis, and P. E. Wellstaed. Using graph theory to visualise piecewise linear systems. In Proceedings 3rd European Control Conference, pages 1631-1636, Rome, Italy, September 1995.

[6] E.D. Sontag. Nonlinear regulation: The piecewise linear approach. IEEE Transactions on Automatic Control, AC-26(2):346-357, April 1981.

[7] R.S. Sreenivas and B.H. Krogh. On condition/event systems with discrete state realizations. Discrete Event Dynamic Systems: Theory and Applications, 1:209235, September 1991. 
[8] J. Stiver, P. J. Ansaklis, and M. D. Lemmon. Interface and controller design for hybrid systems. In P. Antsaklis, W. Kohn, A. Nerode, and S. Sastry, editors, Hybrid Systems II, volume 999 of Lecture Notes in Computer Science, pages 462492. Springer-Verlag, Berlin, 1995. 